\title{
«L’Année stendhalienne» 15
}

\section{Michel Arrous}

\section{(2) OpenEdition}

\section{Journals}

\section{Édition électronique}

URL : http://journals.openedition.org/studifrancesi/10660

DOI : 10.4000/studifrancesi. 10660

ISSN : 2427-5856

\section{Éditeur}

Rosenberg \& Sellier

\section{Édition imprimée}

Date de publication : 1 décembre 2017

Pagination : 560-561

ISSN : 0039-2944

\section{Référence électronique}

Michel Arrous, « «L'Année stendhalienne» 15 », Studi Francesi [En ligne], 183 (LXI | III) | 2017, mis en ligne le 01 février 2018, consulté le 21 janvier 2021. URL : http://journals.openedition.org/ studifrancesi/10660 ; DOI : https://doi.org/10.4000/studifrancesi.10660

Ce document a été généré automatiquement le 21 janvier 2021.

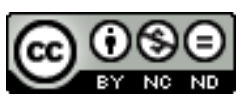

Studi Francesi è distribuita con Licenza Creative Commons Attribuzione - Non commerciale - Non opere derivate 4.0 Internazionale. 


\title{
«L'Année stendhalienne» 15
}

\author{
Michel Arrous
}

\section{RÉFÉRENCE}

«L'Année stendhalienne» 15, Paris, Honoré Champion, 2016, 444 pp.

1 Deux ensembles dans cette copieuse livraison: «Stendhal's many lives», journée d'étude organisée à l'Université de Virginie en septembre 2014, «Stendhal en mouvement», colloque parisien de décembre 2015.

Organisateur du premier, Antoine GUIBAL avait proposé d'examiner la «fièvre biographique» dont Stendhal ne se défit jamais, en se limitant, façon de parler, à Haydn, Napoléon, Rossini et Brulard, œuvres fort disparates de par leur nature et leur visée. Son substantiel avant-propos (pp. 9-14) rappelle l'équivocité du terme «Vie» pour désigner un genre polymorphe. De ses Phares Philippe BERTHIER (pp.15-26) éclaire les premiers débuts dans le genre du jeune Beyle passionné par le panthéon des gloires romaines et françaises que lui découvre le père Ducros. Le désir d'écrire la vie des grands hommes est né en ces instants, mais trop engagé dans de véritables plaidoyers pro domo, Stendhal n'eut jamais l'objectivité ou la neutralité nécessaire à l'historien. D'autre part, alors qu'il n'a cessé de pratiquer le pèlerinage littéraire, il n'a rédigé aucune vie d'écrivain sinon la sienne; il a préféré écrire des vies d'artistes pour les situer dans l'Histoire comme autant de phares qui «éclairent plus loin le cheminement de la caravane humaine». Stendhal aurait-il validé l'aphorisme «Vivre ou écrire, il faut choisir»? Certes, il n'a jamais pensé que la vie devait aboutir ou s'investir dans une œuvre; il s'est plutôt voué à écrire et à vivre, sans pour autant tout écrire, et surtout pas le bonheur. Dans Vies majuscules, vies minuscules (pp. 27-48), c'est d'abord un Stendhal face à Barthes que campe Philippe ROGER. Pour Stendhal qui ne s'en laisse pas conter, le culte du grand homme mérite examen: il y a les fausses gloires et les vraies, celles qui comptent mais dont il n'est pas l'unique narrateur (Haydn, Mozart, l'Empereur) puisqu'il passe non sans désinvolture du plagiat à la compilation. La tactique du narrateur est parfois plus subtile, par exemple quand le Touriste entrelace vérité et 
fiction, sa vie quelque peu déguisée à d'autres fugitives ou minuscules. David F. BELL étudie les liens entre les travaux biographiques et les grands romans des années 1830 (Les "Vies" de Stendhal, ou comment écrire une biographie pour devenir romancier?, pp. 49-64) en prenant appui sur la biographie de Napoléon (1817-1818, 1836-1837), nouveau modèle du rapport entre l'individu et l'Histoire. La méthode du biographe dans les Mémoires sur Napoléon, notamment les intrusions et commentaires du narrateur, rappelle les caractéristiques narratives du Rouge et de la Chartreuse, proximité renforcée par les intersections bien connues entre le roman de 1839 et le chapitre VIII des Mémoires. Écrire de soi comme d'un autre, lire d'un autre comme de soi (pp.65-85): Jacques NEEFS, qui revient sur la rupture provoquée par la Vie de Henry Brulard dans la tradition des «Vies», examine la trame initiale du manuscrit et les ajouts interlinéaires afin de «mesurer les gestes de l'écriture de soi se traçant comme récit d'un autre», sans négliger l'entrelacs du récit, des plans et dessins qui sont autant de localisation de soi. Le procédé implique tant le lecteur qu'il devient HB.

3 Le programme du colloque «Stendhal en mouvement» proposait d'envisager un Stendhal en partance, en quelque sorte un nomade dans l'espace des voyages aussi bien que dans celui des arts. L'historien maritimiste Nicolas COCHARD suit Stendhal dans les ports (pp. 91-106). Le Touriste, «terrien sur la côte», que dit-il de la modernisation de l'univers maritime? À vrai dire, Stendhal ne s'enthousiasme guère pour le transport maritime; néanmoins l'univers et la société portuaires l'intéressent et les Mémoires d'un touriste seraient sur ce point un régal pour l'historien du XIXe siècle. Yves ANSEL a entrepris de récrire le décevant article «Voyages» du Dictionnaire amoureux de D. Fernandez, sur l'air connu de Voyages, voyages... (pp. 107-131). Partir, ne pas s'attacher, voir du nouveau et connaître les hommes (sans aller jusqu'en Australie ou Ceylan), c'était le principe vital de Stendhal à qui le mouvement était nécessaire, même s'il n'a pas rapporté la totalité de ses expériences, et l'on pense à la plus terrible, la campagne de 1812. Évoquer Stendhal en déplacement impose qu'on suive son marchand de fer à la découverte de la France de Louis-Philippe. Dans ce récit apparemment sans ordre ni stratégie, Serge LINKÈs repère les rapports formels entre journal de voyage et journal intime, entre le procès-verbal de la réalité historique et la mise en fiction (Pour une poétique touristique: Stendhal et la mise en récit du voyage, pp. 133-150), contribution que complètent celles de Nicolas ALLARD (Anecdotes mouvantes et mouvance des anecdotes, pp. 239-26) et de Karin GUNDERSEN (Les déclencheurs du voyage dans le temps chez Stendhal autobiographe, pp. 265-271). L'approche de Laure LEVÊQUE (L'échappée Beyle. Du paysage au pays: les "Voyages en France" à l'appui d'une reterritorialisation, pp. 151-167) prend en compte la subjectivité du Touriste «en quête de la région où vivre», dans une France en voie d'uniformisation à laquelle on échappe par ces «moments de plénitude» qu'offre la contemplation de certains paysages. La dynamique propre au texte stendhalien, Antoine GUiBAL la perçoit chez le biographe de Rossini, un Rossini en mouvements (pp. 169-192). Dans la biographie de 1823, farcie de digressions et d'interventions de l'auteur dont le goût musical fluctue, se lisent un manifeste romantique contre la rigueur et ses règles ainsi qu'un éloge de la vivacité d'imagination chez ce musicien «rarement sublime». Puisqu'il s'agit de musique, qu'en est-il du mouvement à proprement parler, demande Béatrice DIDIER qui rappelle le principe énoncé par Stendhal parlant de la Gazza ladra: «Le mouvement fait tout pour l'expression» (pp.193-202). Partisan d'une esthétique de l'expression Stendhal est surtout sensible au rapport entre vitesse et signification, notamment à l'opéra, bien que 
la voix soit moins rapide que l'instrument. Forme subtile du mouvement, l'attirance ou le fluide spirituel de la sympathie des âmes à l'unisson, phénomène d'intersubjectivité que Lydia BAUER interprète en comparatiste ("Les Affinités électives": flux et reflux de la sympathie chez Rousseau, Goethe et Stendhal, pp. 203-215). Il suffit de penser aux scènes de rencontre ou à la belle image des deux anges exilés parmi les hommes. Autre forme de mouvement, celle de la temporalité ou du fil de l'Histoire. Marie-Rose CORREDOR en évalue les différents régimes ("La Chartreuse de Parme": "feuilletages» du temps, pp. 217-228). Dans ce roman, la relation au temps est instable et le devenir incertain: «À travers Fabrice, l'Histoire ne peut ni advenir ni disparaître, mais on ne peut faire qu'elle n'ait pas eu lieu». Afin de surprendre le diariste dans le mouvement de sa pensée et de son écriture, Philippe BERTHIER invite à délaisser l'édition de V. Del Litto qui distinguait artificiellement «journal élaboré» et «journal brut» pour le surprenant «fatras» (Beyle dixit) des premiers cahiers tels qu'on peut les lire depuis 2013 (Mobile / Stabile: la cinétique paradoxale des "Journaux et papiers", pp. 229-237). Sans doute pour ne pas diminuer la vitesse de l'écriture, Stendhal a oublié ou sacrifié la ponctuation dans ses manuscrits autobiographiques; en revanche, il a usé voire abusé des signes de «surponctuation» que sont le soulignement, la majuscule, l'italique et la parenthèse que Fabienne BERCEGOL examine en reprenant et prolongeant l'analyse par S. Sérodes de ses diverses fonctions dans le déroulement syntaxique ( $A u$ détour du récit: les parenthèses dans la "Vie de Henry Brulard", pp. 273-300). Loin d'occuper une place secondaire dans l'autobiographie - plus de 500 occurrences! - ce procédé en dit long sur la posture énonciative de Stendhal. On a affaire à une intrusion d'auteur qui prend de multiples formes: explication-traduction, définition, rappel opportun, citation ou parole rapportée, mise en relief, aparté, rétrospection. Avec «De premier mouvement». Sentiment et mouvement chez Stendhal (pp. 301-324), Xavier BOURDENET vise la représentation du sentiment amoureux que Stendhal comprend comme un mouvement qui emporte l'individu, par exemple lors de la cristallisation ou dans son évolution, selon une cinétique alternant statisme et mobilité. Le goût de l'ailleurs et le goût de l'autre définissant Stendhal, on a pu très tôt le voir en adepte du vagabondage en tout genre tant son existence a connu de scansions. Béatrice DIAZ le voit en auteur soucieux d'échapper par tous les moyens au statut d'écrivain professionnel, de se séparer de la "canaille écrivante», sans omettre le recours à l'alibi ou à la mise en scène par le biais d'avertissements et de préfaces quand il s'adresse au public. Là où la critique contemporaine n'a vu la plupart du temps qu'une pose, on perçoit un mouvement grâce aux bien nommés Passages de Stendhal (pp. 325-343).

4 Dans le premier des «Varia», Maxime triquenaux, plutôt que de se demander si Stendhal fut féministe, considère les relations de genre à un niveau global, à partir du concept de masculinité («L'illustre et vaste corporation à laquelle j'ai l'honneur d'appartenir». Rapports de genre et masculinités dans la première partie du "Rouge et le Noir", pp. 347-367). Quoique limité, le corpus choisi permet de mesurer la masculinité «hégémonique» de Julien (la femme objet de conquête) chez qui pointe la «tentation de la misogynie». Dans le second, Suzanne MILDNER-ARDISSON propose Mina et Féder: des "amoureux à la Werther» revus par Stendhal (pp. 369-383). L'amour à la Werther, le plus pur des amours, est-il possible de le vivre réellement, selon la thèse soutenue dans De l'Amour?

5 Les «Documents» contiennent, publiée et analysée par François BRONNER, Une note inédite de Stendhal en anglais du $1^{\mathrm{er}}$ novembre 1833, sur les affaires d'Espagne; deux lettres inédites de 1836 au général de Cubières et au marquis Giacomo Ricci, publiées et 
commentées par Saverio ALMINI, et des notes explicatives de Jean-Luc BUSSET sur quelques expressions désormais élucidées. En guise d'ultime hommage, saluons Le syndrome de Stendhal, Proust, Dostoïevski, Onfray du regretté Paul DESALMAND (pp. 425-426). 